\title{
Enhancement of the Photochromic Performance of Spirooxazine in Sol-Gel Derived Organic-Inorganic Hybrid Matrices by Additives
}

\author{
L. HOU \\ Institut für Neue Materialien, Geb. 43 Im Stadtwald, D-66123 Saarbrücken, Germany; and \\ Shanghai Institute of Optics and Fine Mechanics, Academia Sinica, 201800 Shanghai, China \\ H. SCHMIDT, B. HOFFMANN AND M. MENNIG \\ Institut für Neue Materialien, Geb. 43 Im Stadtwald, D-66123 Saarbrücken, Germany
}

\begin{abstract}
Organic-inorganic hybrid materials synthesized via sol-gel processing are excellent solid matrices for photochromic dyes like spirooxazine, and the photochromic performance can be further enhanced by introducing suitable additives. This work describes the effects of additives on the photochromic intensity $\left(\Delta A_{0}\right)$, decolouration rate $(k)$ and photostability of spirooxazine in sol-gel derived organic-inorganic hybrid matrices. They include fluoroalkylsilane (FAS), bisphenol $A$ (BPA) and methyl-imidazole (MI). FAS enhances both $\Delta A_{0}$ and photostability, but has little effect on $k$. The higher the content of BPA, the higher the $\Delta A_{0}$ and the higher the photostability, but the lower the $k$. The effect of MI on $\Delta A_{0}$ and $k$ is not so considerable as that of BPA and is a little complicated, while significant improvement in photostability is achieved by the addition of MI at an optimum concentration with the presence of BPA at a higher content.
\end{abstract}

Keywords: organic-inorganic hybrids, sol-gel, photochromic performance, spirooxazine

\section{Introduction}

Organic-inorganic hybrid materials (OIHMs), a new family of porous solid materials synthesized via sol-gel processing have been the subject of extensive research activities since they were first reported by Schmidt and Philipp [1] in 1984. The low-temperature processing method provides a new opportunity to organic dyes to be hosted in solid matrices. The porous structure of these materials offers free spaces for photochromic dyes to undergo reversible structural changes during photochromic transformations. In addition, their rigidity can be tailored by adjusting composition, employing proper precursors and introducing appropriate additives, so that the native structure of the photochromic dye can survive the shrinkage of the gels during drying, necessary heat treatment, and even UV irradiation. The existing publications have shown that the sol-gel derived OIHMs are ideal solid matrices for photochromic dyes such as spirooxazine [2-4], spiropyran [5] and 2,3-diphenylindenone [6], although the photochromic performance needs to be further improved for practical applications. Aiming at enhancing the photochromic performance of spirooxazine (SO), three additives (FAS, BPA and MI) were introduced into the SO-OIHM photochromic system in the present work. The effects of the additives on photochromic intensity $\left(\Delta A_{0}\right)$, decolouration rate $(k)$ and photostability were investigated. The results show an encouraging enhancement of the photochromic performance by rational employment of the additives.

\section{Experimental}

Sols were prepared via the sono sol-gel process described in a previous paper [7] using ethyltriethoxysilane (ETES) and 3-glycidoxypropyltrimethoxysilane 
(GPTMS) as starting precursors at a molar ratio of $3: 7$. Water was added in the form of $0.1 \mathrm{~N} \mathrm{HNO}_{3}$ at a molar ratio of 1.5 to the total silanes. Concentrations of all the additives and the spirooxazine dye, 1,3-dihydro1,3,3-trimethyl-spiro-[2H-indole-2,3'-[3H]-naphth[2, 1-b][1,4]-oxazine (Aldrich Chem. Inc.), are expressed in their molar ratios (MRs) to the total silanes. Dipcoating method was used for the preparation of coatings on microscopic glass slides. After being dried in air for 12-24 hours the coatings were heated at $110^{\circ} \mathrm{C}$ for $2 \mathrm{~h}$. A computer contolled apparatus [7] was used for the measurement of photochromic performance. Photochromic intensity $\left(\Delta A_{0}\right)$ defined as the absorbance at the photostationary state under UV irradiation, and decolouration rate $(k)$ were deduced from the colouring-fading curve. For the evaluation of photostability, the coatings were subjected to UV irradiation at an intensity of $60 \mathrm{~mW} / \mathrm{cm}^{2}$. The variation of $\Delta A_{0}$ with UV irradiation time $(\tau)$ up to $60 \mathrm{~h}$ was monitored and relative photochromic intensity, $\Delta A^{\tau} / \Delta A_{0}$, was plotted against UV irradiation time. The irradiation hours needed for $\Delta A^{\tau}$ to reach $0.5 \Delta A_{0}, \tau_{0.5}$, was evaluated from the $\Delta A^{\tau} / \Delta A_{0}$ vs $\tau$ curve and is used as a measure of photostability.

\section{Results and Discussion}

Figures 1 and 2 show the effects of BPA and MI on $\Delta A_{0}$ and $k$ of the coatings, respectively. The concentrations of FAS $\left(\mathrm{MR}_{\mathrm{FAS}}\right)$ and the dye $\left(\mathrm{MR}_{\mathrm{SO}}\right)$ are 0.015 and 0.010 , respectively. It can be clearly seen in the figures that the higher the content of BPA, the higher the $\Delta A_{0}$,

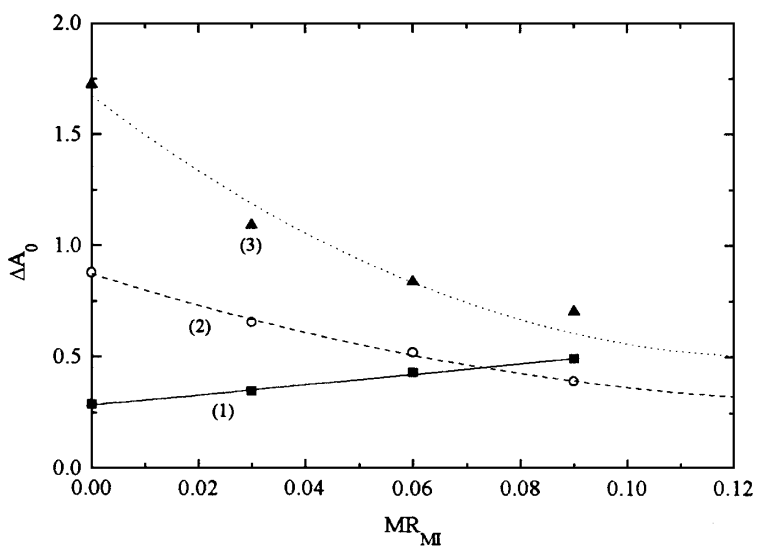

Figure 1. Photochromic intensity $\left(\Delta A_{0}\right)$ as a function of MI concentration $\left(\mathrm{MR}_{\mathrm{MI}}\right)$ at different $\mathrm{BPA}$ content $\left(\mathrm{MR}_{\mathrm{BPA}}\right)$ : (1) 0, (2) 0.15, (3) 0.30 .

\begin{tabular}{lr}
$\begin{array}{l}\text { Table 1. } \\
\text { ferent content of BPA. }\end{array}$ \\
\hline MR $_{\text {BPA }}$ & $\tau_{0.5}(\mathrm{~h})$ \\
\hline 0.05 & 12.8 \\
0.15 & 18.5 \\
0.20 & 31.5 \\
0.25 & 49.8 \\
\hline
\end{tabular}

and the lower the $k$. The effect of MI on $\Delta A_{0}$ and $k$, however, is not so considerable as that of BPA and is a little complicated. When there is no BPA a slow increase in $\triangle A_{0}$ is shown with increasing concentration of MI, while for the BPA-containing coatings $\Delta A_{0}$ decreases with increasing concentration of $\mathrm{MI}$, and the higher the content of BPA, the faster is the decrease of $\Delta A_{0}$. On the other hand, it is evident in Fig. 2 that $k$ increases with increasing concentration of MI when the content of BPA is equal to 0.30 and decreases with increasing concentration of MI when no BPA is used, though the variations of $k$ in both cases are very slow. It is worth noting that MI exhibits little influence on $k$ when the content of BPA equals 0.15.

The significant enhancement of photostability by BPA is undoubtedly demonstrated by the monotonic increase of $\tau_{0.5}$ with increasing content of BPA in Table 1 . The effcet of MI on photostability is depicted in Fig. 3 by plotting $\tau_{0.5}$ against $\mathrm{MR}_{\mathrm{MI}} \cdot \tau_{0.5}$ goes through a maximum with increasing $\mathrm{MR}_{\mathrm{MI}}$, suggesting an optimum concentration of MI around 0.05 .

In order to achieve high photostability it is necessary to have both a high $\Delta A_{0}$ before UV irradiation

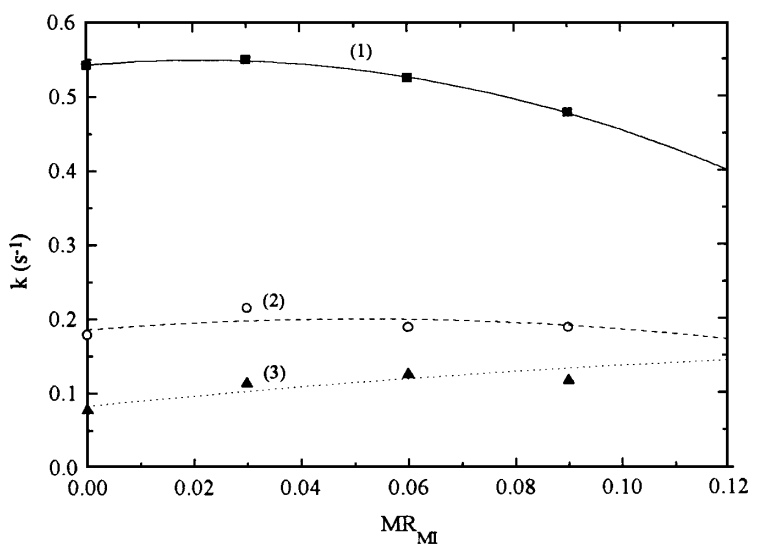

Figure 2. Decolouration rate $(k)$ as a function of MI concentration $\left(\mathrm{MR}_{\mathrm{MI}}\right)$ at different BPA content $\left(\mathrm{MR}_{\mathrm{BPA}}\right)$ : (1) 0, (2) 0.15, (3) 0.30 . 


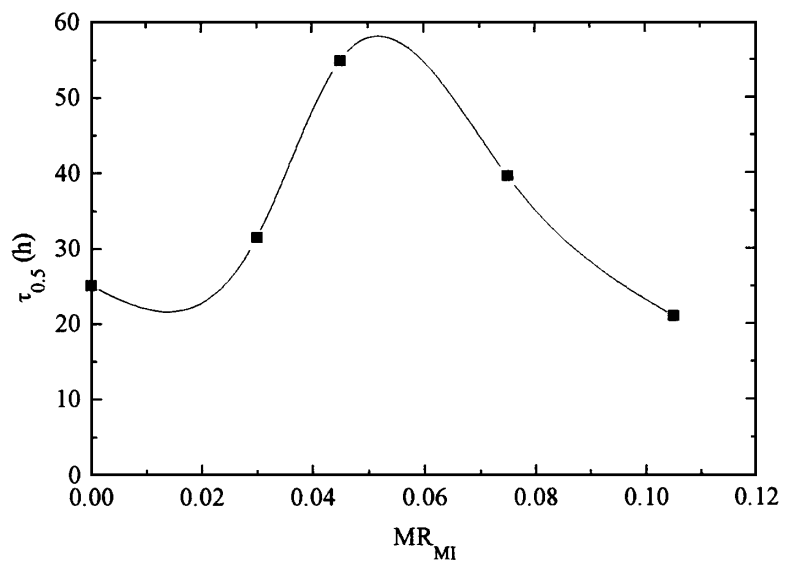

Figure 3. Variation of $\tau_{0.5}$ with MI concentration (MR $\mathrm{MI}_{\mathrm{MI}}$ ) at a BPA content $\left(\mathrm{MR}_{\mathrm{BPA}}\right)$ of 0.20 .

and a long $\tau_{0.5}$ against UV irradiation. There are two kinds of factors affecting $\Delta A_{0}$ and $\tau_{0.5}$ : electric and steric. In general, the higher the polarity of the environment in the matrix, the higher the thermal stability of the colored form, implying a higher $\Delta A_{0}$ and a lower $k$. Addition of phenol compounds, which contain aromatic and $\mathrm{OH}$-groups such as BPA, helps effectively stabilize the coloured form. The enhancement of $\Delta A_{0}$ and retardation of $k$ are due to the electric effect of BPA as it creates an environment with higher concentration of OH-groups.

The steric effect exerted by matrix on $\Delta A_{0}$ and photostability is contradictory: a high matrix rigidity always suppresses $\Delta A_{0}$ whereas it is favourable not only to the stability of the matrix itself but also to the protection of the dye molecules from UV irradiation. Therefore, it is advisable to optimize the matrix rigidity, taking both $\Delta A_{0}$ and $\tau_{0.5}$ into account. MI is thought to act as a catalyst for the hydrolysis-condensation of the silanes and for the polycondensation between BPA and GPTMS, and as an initiator for the polymerization of the epoxy groups in GPTMS. The interaction between the silanes, BPA and MI results in the complicated effect of MI in the presence of BPA as shown in Figs. 1-3. The cooperative effect of BPA and MI leads to an optimum concentration of MI (ca 0.05) where the best photostability is achieved.

In a previous paper [4] we reported that the introduction of a small concentration of FAS leads to significant increase in both the photochromic intensity and the photostability of spirooxazine in OIHM coatings but has no influence on the decolouration rate. This is also true when BPA and MI co-exist with FAS in the coatings. The higher the concentration of FAS, the higher the $\Delta A_{0}$ and the photostability. $\tau_{0.5}$ for the coatings with FAS at the concentrations of $0,0.009$ and 0.015 are $21.5 \mathrm{~h}, 25.5 \mathrm{~h}$, and $31.5 \mathrm{~h}$, respectively. The reason for the enhancement is that the enrichment of fluorine in FAS at the surface layer of the coating results in a non-stick surface due to the low surface energy. This non-stick surface prevents water, oil and other compounds from clinging onto and penetrating into the coating. These compounds themselves may be harmful to the photochromic dye or under UV irradiation they may generate new substances that are detrimental to the dye.

\section{Conclusions}

Great enhancement of the photochromic performance of spirooxazine in organic-inorganic hybrid matrices has been achieved by rational employment of BPA, MI and FAS as additives. BPA serves to maintain a high photochromic intensity, MI helps optimize the matrix rigidity and bulk protection of the dye, and FAS contributes to the surface modification so as to prevent harmful compounds from entering the coatings. In most cases the decolouration rate is sufficiently high $\left(>0.1 \mathrm{~s}^{-1}\right)$ and is not a problem to practical applications.

\section{References}

1. H. Schmidt and G. Philipp, J. Non-Cryst. Solids 63, 283 (1984).

2. L. Hou, B. Hoffmann, M. Mennig, and H. Schmidt, J. Sol-Gel Sci. Techn. 2, 635 (1994).

3. L. Hou, M. Mennig, and H. Schmidt, Chimica Chronica, New Series 23, 175 (1994).

4. L. Hou, M. Mennig, and H. Schmidt, Proc. SPIE (1994), vol. 2255 , p. 26.

5. D. Levy, S. Einhorn, and D. Avnir, J. Non-Cryst. Solids 113, 137 (1989).

6. S.A. Yamanaka, J.I. Zink, and B. Dunn, Proc. SPIE (1992), vol. 1758, p. 372.

7. L. Hou, M. Mennig, and H. Schmidt, Proc. SPIE (1994), vol. 2288, p. 328. 